\title{
Crystal structure of $\left(\eta^{5}\right.$-pentamethylcyclopentadienyl) $\left(\eta^{5}\right.$-1-(2,2-dime- thylpropyl)cyclopentadienyl)titaniumdichloride, $\mathrm{C}_{20} \mathrm{H}_{30} \mathrm{Cl}_{2} \mathrm{Ti}$
}

\author{
J. Stroot, D. Haase, W. Saak and R. Beckhaus* \\ Carl von Ossietzky Universität Oldenburg. Fachbereich Chemie. PF 2503. D-2611 I Oldenburg. Germany
}

Received September 13, 2001, accepled December 12, 2001: CCDC-No. 1267/728

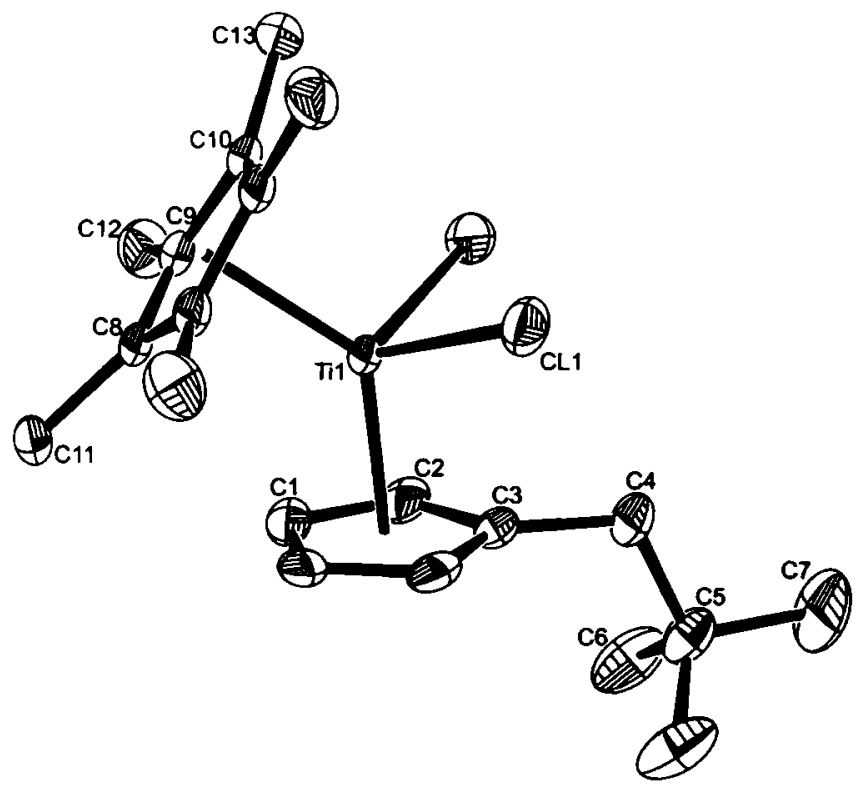

exocyclic carbon atom of the fulvene ligand [4] with $\mathrm{HCl}$.

The crystal structure of the reaction product shows the metal atom residing on a crystallographic mirror plane bisecting the two staggered cyclopentadienyl rings.

Table 1. Data collection and handling.

\begin{tabular}{ll} 
Crystal: & red needle, size $0.10 \times 0.10 \times 0.70 \mathrm{~mm}$ \\
Wavelength: & Mo $K_{\alpha}$ radiation $(0.71073 \AA)$ \\
$\mu:$ & $6.86 \mathrm{~cm}^{-1}$ \\
\hline Diffractometer, scan mode: & Stoe IPDS, 136 exposures, $\Delta \varphi=1.4^{\circ}$ \\
$2 \theta_{\text {max: }}$ & $52.06^{\circ}$ \\
$N(h k l)_{\text {measured, }} N(h k l)_{\text {unique }}:$ & 14106,1909 \\
Criterion for $I_{\text {obs, }} N(h k l)_{\text {gl }}:$ & $I_{\text {obs }}>2 \sigma\left(I_{\text {obs }}\right), 1286$ \\
$N(\text { param })_{\text {refined: }}$ & 175 \\
Programs: & SHELXS-97 [5], SHELXL-97 [6] \\
& ORTEP-3 [7]
\end{tabular}

\section{Abstract}

$\mathrm{C}_{20} \mathrm{H}_{30} \mathrm{Cl}_{2} \mathrm{Ti}$, orthorhombic, Pnma (No. 62), $a=14.3763(8) \AA$, $b=12.105(1) \AA, c=11.6138(9) \AA, V=2021.0 \AA^{3}, Z=4$, $R_{\mathrm{gt}}(F)=0.032, w R_{\mathrm{ref}}\left(F^{2}\right)=0.062, T=193 \mathrm{~K}$.

\section{Source of material}

An excess of $\mathrm{HCl}$-gas ( $99.9 \%$, Aldrich) was bubbled in a solution of $1.764 \mathrm{~g}(5.0 \mathrm{mmol})$ of $\mathrm{Cp}^{*} \mathrm{Ti}\left(\eta^{6}-\mathrm{C}_{5} \mathrm{H}_{4} \mathrm{C}(\mathrm{H})^{\prime} \mathrm{Bu}\right) \mathrm{Cl}$ in $20 \mathrm{ml}$ of THF at ambient temperature. The colour of the reaction mixture changed immediately from green to red. After stirring the solution at $298 \mathrm{~K}$ for an additional $0.5 \mathrm{~h}$, the solvent was removed in vacuo and the product was recrystallized from $n$-hexane $(10 \mathrm{ml})$. By fractionated crystallization, the suitable crystals for diffraction are obtained at $273 \mathrm{~K}$, crystallization at $213 \mathrm{~K}$ yields $1.654 \mathrm{~g}$ (85\%) $\mathrm{Cp}^{*}\left(\eta^{5}-\mathrm{C}_{5} \mathrm{H}_{4} \mathrm{CH}_{2} \mathrm{C}\left(\mathrm{CH}_{3}\right)_{3}\right) \mathrm{TiCl}_{2}$.

\section{Discussion}

It is well established that replacement of an $\eta^{5}$-cyclopentadienyl ligand by an $\eta^{5}$-pentamethylcyclopentadienyl ligand in transition metal complexes results in significant changes in reactivity, stability and other properties, owing to both steric as well as to electronic effects that accomplish replacement of the hydrogen atoms by methyl groups [1-3]. In this context use of monosubstituted $\eta^{5}$-cyclopentadienyl ligands and their effects on structural properties are of high interest. Bent-metallocenes with one monosubstituted $\eta^{5}$-cyclopentadienyl ligand are easily available by reaction of pentafulvene titanium complexes substituted at the

Table 2. Atomic coordinates and displacement parameters (in $\AA^{2}$ ).

\begin{tabular}{llllrl}
\hline Atom & Site & $x$ & $y$ & \multicolumn{1}{c}{$z$} & \multicolumn{1}{l}{$U_{\text {iso }}$} \\
\hline H(1) & $8 d$ & $1.015(2)$ & $0.352(2)$ & $0.255(2)$ & $0.031(6)$ \\
H(2) & $8 d$ & $0.910(2)$ & $0.414(2)$ & $0.409(2)$ & $0.036(7)$ \\
H(4) & $8 d$ & $0.780(2)$ & $0.185(2)$ & $0.546(2)$ & $0.031(6)$ \\
H(6A) & $8 d$ & $0.894(3)$ & $0.422(3)$ & $0.674(3)$ & $0.09(1)$ \\
H(6B) & $8 d$ & $0.985(3)$ & $0.351(3)$ & $0.626(3)$ & $0.09(1)$ \\
H(6C) & $8 d$ & $0.966(2)$ & $0.355(2)$ & $0.762(3)$ & $0.068(9)$ \\
H(7A) & $8 d$ & $0.759(4)$ & $0.186(4)$ & $0.754(4)$ & 0.130 \\
H(7B) & $4 c$ & $0.833(5)$ & $1 / 4$ & $0.835(6)$ & 0.130 \\
H(11A) & $8 d$ & $1.053(2)$ & $0.189(2)$ & $0.070(2)$ & $0.062(9)$ \\
H(11B) & $4 c$ & $1.024(3)$ & $1 / 4$ & $-0.035(4)$ & $0.05(1)$ \\
H(12A) & $8 d$ & $0.948(3)$ & $0.472(2)$ & $0.093(2)$ & $0.050(9)$ \\
H(12B) & $8 d$ & $0.853(2)$ & $0.503(2)$ & $0.117(2)$ & $0.048(8)$ \\
H(12C) & $8 d$ & $0.879(2)$ & $0.491(2)$ & $-0.011(3)$ & $0.06(1)$ \\
H(13A) & $8 d$ & $0.685(3)$ & $0.445(3)$ & $0.073(3)$ & $0.07(1)$ \\
H(13B) & $8 d$ & $0.627(3)$ & $0.352(3)$ & $0.062(3)$ & $0.07(1)$ \\
H(13C) & $8 d$ & $0.678(2)$ & $0.396(2)$ & $-0.043(3)$ & $0.065(9)$ \\
& & & & & \\
\hline
\end{tabular}

\footnotetext{
* Correspondence author

(e-mail: ruediger.beckhaus@uni-oldenburg.de)
} 
Table 3. Atomic coordinates and displacement parameters (in $\AA^{2}$ ).

\begin{tabular}{|c|c|c|c|c|c|c|c|c|c|c|}
\hline Atom & Site & $x$ & $y$ & $z$ & $U_{11}$ & $U_{22}$ & $U_{33}$ & $U_{12}$ & $U_{13}$ & $U_{23}$ \\
\hline $\mathrm{Ti}(1)$ & $4 c$ & $0.83053(4)$ & $1 / 4$ & $0.24591(4)$ & $0.0177(3)$ & $0.0283(3)$ & $0.0125(2)$ & 0 & $0.0013(2)$ & 0 \\
\hline $\mathrm{Cl}(1)$ & $8 d$ & $0.72965(5)$ & $0.10689(5)$ & $0.30347(5)$ & $0.0351(4)$ & $0.0427(3)$ & $0.0258(3)$ & $-0.0100(3)$ & $0.0022(3)$ & $0.0077(2)$ \\
\hline$C(1)$ & $8 d$ & $0.9793(2)$ & $0.3085(2)$ & $0.3011(2)$ & $0.022(2)$ & $0.070(2)$ & $0.021(1)$ & $-0.013(1)$ & $-0.005(1)$ & $0.001(1)$ \\
\hline$C(2)$ & $8 d$ & $0.9221(2)$ & $0.3431(2)$ & $0.3915(2)$ & $0.033(2)$ & $0.044(2)$ & $0.020(1)$ & $-0.007(1)$ & $-0.009(1)$ & $-0.003(1)$ \\
\hline$C(3)$ & $4 c$ & $0.8854(3)$ & $1 / 4$ & $0.4484(2)$ & $0.026(2)$ & $0.045(2)$ & $0.015(2)$ & 0 & $-0.005(1)$ & 0 \\
\hline$C(4)$ & $4 c$ & $0.8226(3)$ & $1 / 4$ & $0.5506(2)$ & $0.036(3)$ & $0.053(2)$ & $0.017(2)$ & 0 & $0.003(2)$ & 0 \\
\hline$C(5)$ & $4 c$ & $0.8739(3)$ & $1 / 4$ & $0.6688(3)$ & $0.052(3)$ & $0.049(2)$ & $0.018(2)$ & 0 & $-0.002(2)$ & 0 \\
\hline C(6) & $8 d$ & $0.9341(3)$ & $0.3521(3)$ & $0.6821(3)$ & $0.093(3)$ & $0.047(2)$ & $0.034(2)$ & $-0.008(2)$ & $-0.021(2)$ & $-0.003(1)$ \\
\hline$C(7)$ & $4 c$ & $0.7992(5)$ & $1 / 4$ & $0.7622(4)$ & $0.090(5)$ & $0.152(6)$ & $0.017(2)$ & 0 & $0.012(2)$ & 0 \\
\hline$C(8)$ & $4 c$ & $0.9174(2)$ & $1 / 4$ & $0.0672(2)$ & $0.020(2)$ & $0.042(2)$ & $0.011(1)$ & 0 & $0.002(1)$ & 0 \\
\hline$C(9)$ & $8 d$ & $0.8586(2)$ & $0.3445(2)$ & $0.0700(2)$ & $0.025(2)$ & $0.035(1)$ & $0.0146(9)$ & $-0.001(1)$ & $0.0033(8)$ & $0.0039(8)$ \\
\hline$C(10)$ & $8 d$ & $0.7651(2)$ & $0.3078(2)$ & $0.0608(2)$ & $0.023(2)$ & $0.041(1)$ & $0.0124(9)$ & $0.002(1)$ & $0.0028(9)$ & $0.0025(8)$ \\
\hline$c(11)$ & $4 c$ & $1.0193(3)$ & $1 / 4$ & $0.0429(3)$ & $0.020(3)$ & $0.057(3)$ & $0.021(2)$ & 0 & $0.005(1)$ & 0 \\
\hline$C(12)$ & $8 d$ & $0.8879(3)$ & $0.4627(2)$ & $0.0669(3)$ & $0.040(2)$ & $0.036(2)$ & $0.045(2)$ & $-0.003(1)$ & $0.006(1)$ & $0.009(1)$ \\
\hline$C(13)$ & $8 d$ & $0.6838(2)$ & $0.3801(3)$ & $0.0361(2)$ & $0.027(2)$ & $0.058(2)$ & $0.030(1)$ & $0.012(1)$ & $0.003(1)$ & $0.010(1)$ \\
\hline
\end{tabular}

Acknowledgment. We thank the Bundesministerium für Bildung und Forschung (03C0276G/4).

\section{References}

1. Clearfield, A; Warner, D. K.; Saldarriaga-Molina, C. H.; Ropal, R.: Structural Studies of $\left(\pi-\mathrm{C}_{5} \mathrm{H}_{5}\right)_{2} \mathrm{MX} \mathbf{X}_{2}$ Complexes and their Derivates. The Structure of Bis( $\pi$-cyclopentadienyl)titanium Dichloride. Can. J. Chem. 53 (1975) 1623-1629.

2. McKenzie, T.; Sanner, R. D.; Bercaw, J. E.: The Crystal and Molecular Structure of Bis(pentamethylcyclopentadienyl)dichlorotitanium (IV). J. Organomet. Chem. 102 (1975) 457-466.

3. Rogers, R. D.; Benning, M. M.; Kurihara, L. K.: The Formation and Crystal and Molecular Structures of ( $\eta^{5}$-Pentamethylcyclopentadienyl) ( $\eta^{5}$-cyclopentadienyl)dichloro-titanium, -zirconium and -hafnium. J. Organomet. Chem. 293 (1985) 51-60.
4. Beckhaus, R.; Lützen, A.; Haase, D.; Saak, W.; Stroot, J.; Becke, S.; Heinrichs, J.: Ein neuartiger Zugang zu Fulvenkomplexen des Titans Diastereoselektive Komplexierung von Pentafulvenen an Cyclopentadienyltitan-Fragmente. Angew. Chem. 113 (2001) 2112-2115; Angew. Chem. Int. Ed. Engl. 40 (2001) 2056-2058.

5. Sheldrick, G. M.: Phase Annealing in SHELX-90: Direct methods for Larger Structure. Acta Crystallogr. A46 (1990) 467-473.

6. Sheldrick, G. M.: SHELXL-97. A Program for Refining Crystal Structures. University of Göttingen, Germany 1997.

7. Farrugia, L. J.: ORTEP-3 for Windows - a version of ORTEP-III with Graphical User Interface (GUI). J. Appl. Crystallogr. 30 (1997) 565. 